\title{
Modelo de Tony Booth y Mel Ainscow para fortalecer la educación inclusiva en educación religiosa
}

\section{Tony Booth and Mel Ainscow's Model for Strengthening Inclusion in Religious Education}

DOI: $10.46932 /$ sfjdv2n5-139

Received in: Oct 1st, 2021

Accepted in: Dec 30th, 2021

\author{
Susan Joy Agurto-Agurto \\ Universidad César Vallejo, Piura, Perú.
}

Magister en Psicología Educativa, Profesora de Ciencias Sociales, Filosofía y Religión. Dirección: A.H. Túpac Amaru I Calle Holanda Mz G3 Lt 21 Distrito 26 de Octubre - Piura -Perú

\section{Mario Napoleón}

Doctor en Educación. Licenciado de Filosofía y Ciencias Sociales. Universidad César Vallejo, Piura, Perú. ORCID: 0000-0001-9494-0850.

Dirección: El Indio C-5 Lote 10-Castilla-Piura-Perú.

E-mail: mbrionesm@ucv.edu.pe

\section{Briones Mendoza}

Doctor en Educación. Licenciado de Filosofía y Ciencias Sociales. Universidad César Vallejo, Piura, Perú.

Dirección: El Indio C-5 Lote 10-Castilla-Piura-Perú.

\section{RESUMEN}

La inclusión educativa está orientado desde el enfoque humanista, siendo necesario darle un sentido desde la práctica de la fe, por lo cual en este estudio se realizó un análisis de la inclusión según el modelo de Tony Booth y Mel Ainscow, a fin de fortalecer el área de educación religiosa, promotora de la educación inclusiva, el cual, tuvo como propósito determinar los aspectos desarrollados en el modelo de Tony Booth y Mel Ainscow que se pueden considerar en el desarrollo de una teoría que sustente la educación inclusiva en dicha área pedagógica. El estudio es de tipo cualitativo y el diseño de la investigación corresponde a la teoría fundamentada, utilizándose como técnica la entrevista individual estructurada, y como instrumento la guía de entrevista estructurada y el cuestionario con opción de respuesta fundamentada. Además, se aplicó la técnica del análisis documental con el instrumento ficha de análisis documental. Se obtuvo como resultado la propuesta de un modelo teórico, a partir del modelo de Tony Booth y Mel Ainscow, de la información recabada de los docentes, del análisis documental y de la espiritualidad del área, concluyendo que el área de Educación Religiosa brinda una formación integral de la persona, pues, los docentes de esta área fomentan un mundo justo y solidario, donde los estudiantes gocen del bienestar y de una educación inclusiva de calidad en las Instituciones Educativas.

Palabras clave: Culturas inclusivas, prácticas inclusivas, educación Inclusiva, políticas inclusivas y modelo INDEX

\footnotetext{
ABSTRACT

In this study, an analysis of the model of Tony Booth and Mel Ainscow was carried out, in order to strengthen the area of religious education, promoting inclusive education, which was intended to
} 
determine the aspects developed in the model of Tony Booth and Mel Ainscow that can be considered in the development of a theory that supports inclusive education in the area of Religious Education. The study is qualitative and the research design is grounded theory, the structured individual interview technique was used, using the structured interview guide and the questionnaire with a grounded answer option as instruments. In addition, the document analysis technique was applied with the document analysis sheet instrument. The result was a proposal for a theoretical model, taken from the theoretical model of Tony Booth and Mel Ainscow, from the information collected from the teachers, from the documentary analysis and from the spirituality of the area, noting that the area of Religious Education provides training integral of the person, therefore, teachers in this area promote a fair and supportive world, where students enjoy well-being and an inclusive quality education in Educational Institutions.

Keywords: Inclusive cultures, develop inclusive practices, inclusive education, develop inclusive policies and Tony Booth and Mel Ainscow model

\section{INTRODUCCIÓN}

En este estudio se realizó el análisis de los aportes del Índice para la inclusión (INDEX) con la finalidad de realizar un aporte en el ámbito de la inclusión educativa en el área de Educación Religiosa. De acuerdo a Tony Booth y Mel Ainscow (2000) la inclusión no es sólo que los estudiantes con discapacidad accedan a las escuelas de la educación básica, sino también disminuir las distintas barreras que les dificultan el aprendizaje y promover la integración de todos los estudiantes. Ya que como se observa, muchos estudiantes experimentan dificultades debido a que no se tienen en cuenta sus características en los procesos de enseñanza y aprendizaje, por lo cual es necesario que dentro del área de Educación Religiosa se considere la necesidad de mejorar la atención a la inclusión educativa.

En Latinoamérica se presentan diversas dificultades, pues, mientras que en unos países es obligatorio ingresar al sistema educativo desde los 3 y 7 años a la educación primaria, en muchos casos hay deficiencias para incluir a los estudiantes que tienen necesidades educativas especiales. (UNESCO, 2014) Además, según Tony Booth y Mel Ainscow (2000) en América Latina existen altos niveles de iniquidad, exclusión y fragmentación social. A pesar del gran desarrollo de la educación, todavía encontramos muchas desigualdades educativas en los aspectos socioeconómicos, culturales y en relación a las características individuales del alumnado, debido al modelo homogeneizador del sistema educativo. Así mismo, el Ministerio de Educación en Perú ha ido mejorando la accesibilidad a la educación para los estudiantes con necesidades educativas especiales, un aspecto positivo y alentador.

Desde el 2015 el MINEDU reconoció el esfuerzo de las instituciones educativas que fomentaban la inclusión denominándolas Escuelas Valora (Jacinto, 2019), aunque estos esfuerzos son mínimos en consideración a la gran necesidad que se presenta a nivel nacional, ya que últimamente en Perú se han implementado muchas estrategias para el desarrollo de la educación inclusiva en las instituciones educativas, no obstante, aún tenemos muchos estudiantes con diferentes necesidades educativas que no 
pueden acceder a la educación y por otro lado, hay muchos niños y niñas que a pesar de formar parte de una institución, no son considerados y no se realizan adaptaciones según sus necesidades de aprendizaje. (Subiría, 2019). La educación piurana está impregnada de diversas barreras como la desinformación y los estereotipos que no permiten promover la educación inclusiva. Es necesario que el Estado garantice el acceso universal a una educación integral y de calidad que brinde al educando la posibilidad de ser una persona independiente y autónoma. (Educación inclusiva Piura, 2011).

En ese sentido, es imperativo mejorar el desarrollo de la educación inclusiva en dicha área, de tal modo que se plantea la pregunta de investigación: ¿Qué aspectos desarrollados en el modelo de Tony Booth y Mel Ainscow se pueden considerar en el desarrollo de una teoría que sustente la educación inclusiva en el área de educación religiosa? Teniendo en cuenta lo dicho, se presenta el objetivo general: Determinar los aspectos desarrollados en el modelo de Tony Booth y Mel Ainscow que se pueden considerar en el desarrollo de una teoría que sustente la educación inclusiva en el área de educación religiosa.

Este estudio es relevante, pues, se busca posicionar la necesidad de una educación inclusiva para favorecer la atención en las necesidades educativas de los estudiantes en un sistema escolar con mucha diversidad, con este estudio se pretende realizar un análisis del modelo de Tony Booth y Mel Ainscow a fin de fortalecer el área de educación religiosa, promoviendo la educación inclusiva, El aporte teórico de este estudio es renovar la perspectiva teórica con que se aprecia la educación inclusiva desde las posturas de los autores investigados y la opinión de los actores directos como son los docentes, en nuestro caso, del área de educación religiosa. Asimismo, es un tema de actualidad, pues según UNESCO (2014) una educación de calidad también tiene como indicador el nivel de inclusión que se realiza en sus ambientes educativos. Como se puede apreciar, la importancia de esta investigación consiste en el aporte de un soporte teórico científico a la práctica del docente de educación religiosa, que lo lleva a comprometerse con la inclusión educativa en el contexto donde le toca actuar.

\subsection{LA EDUCACIÓN INCLUSIVA}

Los principios epistemológicos que conducen la educación inclusiva son potencialmente aspectos programados para la protección social y la equidad, con la finalidad de que todas las personas desarrollen sus capacidades y potencialidades para obtener una calidad de vida. (Durán y Martínez, 2018). La dignidad de la persona es un principio epistemológico de la educación inclusiva y para la Doctrina Social de la Iglesia es parte intrínseca del hombre y la mujer, porque somos imagen y semejanza de Dios, y ello nos conduce a existir en relación al otro, porque Dios es Padre, es Hijo, es Espíritu Santo, Él es una comunidad de amor y nosotros estamos llamados a vivir esta comunidad de amor con los demás. Es así 
que, el hombre está llamado a promover la dignidad de la persona y su vocación, a alcanzar la calidad de vida en un encuentro solidario con los demás. (Pontificio Consejo "Justicia y Paz", 2005).

El tema de la educación inclusiva es uno de los más importantes a nivel internacional, es por ello que la ONU ha tenido a bien abordar este principio definiéndolo como la adquisición de una educación de calidad, el cual es fundamental para brindar una mejor calidad vida de todas las personas y promover el desarrollo sostenible. En el aspecto de la inclusión de las mujeres en el ámbito educativo, se ha realizado grandes avances, incrementándose el nivel de alfabetización, aunque, aún es necesario realizar más esfuerzos para la consecución de una educación universal. (ONU, 2018).

La UNESCO marca un hito definiendo la educación inclusiva, como el proceso para integrar a los estudiantes con distintas características en la enseñanza tradicional, es un camino que analiza cómo convertir el sistema educativo, con la finalidad de atender la diversidad de los estudiantes. La educación inclusiva tiene el propósito de permitir que los maestros y estudiantes se sientan bien ante la diversidad y no la perciban como un problema, sino como una oportunidad para enriquecer el aprendizaje" (UNESCO, 2008).

\subsection{EL ÍNDICE DE INCLUSIÓN}

En el índice de Booth y Ainscow se describen los valores como guías esenciales para promover la acción inclusiva en la educación. Es importante reconocer los valores que se relacionan con la educación inclusiva, pues, estos serán los parámetros fundamentales para saber qué acciones debemos realizar. Para desarrollar una cultura inclusiva se debe tener en cuenta el desarrollo de valores inclusivos y que estos sean parte de la comunidad educativa, estos valores pertenecientes a la cultura escolar serán los que guíen las acciones que se tengan en cuenta en relación a las políticas escolares de cada institución para apoyar el proceso de desarrollo hacia una escuela inclusiva. (Tony Booth y Ainscow, 2000). El modelo de Tony Booth y Mel Ainscow, se define en un libro denominado: "Guía para la educación inclusiva", el index fue publicado en el año 2000, contando con un equipo que desarrolló distintas experiencias para el desarrollo inclusivo en las instituciones educativas. El index es una guía para la auto-evaluación o diagnóstico de todos los aspectos de una institución educativa, teniendo en cuenta todas las actividades dentro y fuera de las aulas. Se motiva a que toda la comunidad educativa participe en la elaboración de un plan inclusivo. (Booth y Ainscow, 2000).

De acuerdo a la definición en el índex, la inclusión no se refiere sólo al acceso de los estudiantes con discapacidad a las instituciones educativas comunes, sino con minimizar o eliminar las barreras que restringen el aprendizaje, integración y participación de todo el alumnado, pues, muchos estudiantes sienten que no se tienen en cuenta sus diferencias en el proceso de enseñanza y aprendizaje, las cuales, pueden ser por tener diferentes normas, valores, creencias y comportamientos, debido a ser parte de otros 
grupos sociales que en muchas ocasiones no pertenecen a la cultura escolar, lo que puede limitar su integración o conducir a la exclusión y discriminación. (Booth y Ainscow, 2000). Además, el Index for Inclusion es un instrumento muy importante que ayuda a diagnosticar la forma en cómo se encuentra la inclusión en las instituciones educativas, para ello, presenta tres dimensiones, las cuales son: crear culturas inclusivas, crear políticas inclusivas y desarrollar prácticas inclusivas. (Subiria, 2019). En ese sentido, la primera dimensión trata sobre la creación de una comunidad escolar acogedora en la que todos los estudiantes sean valorados y así puedan alcanzar sus logros de aprendizaje, en la segunda dimensión se busca asegurar que la inclusión sea el punto central de desarrollo de la institución educativa, teniendo en cuenta todas las actividades que puedan aportar a la obtención de la inclusión y en la tercera dimensión, el objetivo es asegurar que las actividades escolares promuevan la integración y participación de los estudiantes en su contexto social. (Booth y Ainscow, 2000).

\section{MÉTODOS}

El diseño de esta investigación fue la teoría fundamentada, la cual, tiene como objetivo producir teoría a partir de textos recabados en contextos naturales, es una forma de analizar diversos aspectos de la realidad, para elaborar nuevas teorías de los datos que hayan sido capturados de manera gradual y así conceptualizarlos, en ese sentido, se recabó la información a través de diversas entrevistas a los docentes de Educación Religiosa de ODEC Piura, con la finalidad de registrar sus ideas, conceptos, experiencias y sentimientos en relación a la educación inclusiva, considerando los aportes del modelo de Tony Booth y Mel Ainscow para el análisis bibliográfico respectivo. Así mismo, el eje esencial de la teoría fundamentada es la producción de conceptos sobre temas relevantes para el investigador y la sociedad, requiere de un análisis constante para realizar la construcción de la teoría, de tal modo que, la teoría fundada se cimienta en la comprensión de la realidad desde el punto de vista de los actores de la investigación, esto quiere decir que, se basa conceptualmente en el interaccionismo simbólico. Por lo cual, se obtendrá una nueva teoría que fortalecerá el desarrollo de la educación inclusiva en el área de Educación Religiosa, eso se explica, porque para este tipo de investigación es indispensable comprender profundamente un fenómeno social, pero, teniendo en cuenta la teoría. (Bautista, 2011)

Los participantes de este estudio fueron los docentes del área de Educación Religiosa que pertenecen a ODEC Piura, de los distritos de Piura, Paita y Sechura, quienes en su mayoría tienen el título de educación religiosa, son nombrados y ostentan un posgrado, (Maestría en educación), y si son de otra especialidad, por lo menos han recibido el curso de complementación en la escuela de educación religiosa, (ESER- PIURA). Los docentes de educación religiosa tienen un tiempo de servicio en la docencia de 3 a más de 25 años, donde los participantes, están entre 15 a 30 años. Los docentes de dicha área, están distribuidos en diversos sectores que comprenden a la ODEC Piura: Sector Piura, Sector Bajo Piura, 
Sector Tambogrande y las Lomas, Sector Paita y Sector Castilla. Estos sectores están dirigidos por diversos coordinadores que monitorean y acompañan la labor docente de cada profesor del área, recibiendo diversas capacitaciones en el aspecto profesional-pedagógico de la mano con los coordinadores de cada sector y en el aspecto espiritual, a través del departamento de pastoral educativa de la oficina. Los docentes del área de educación religiosa siguen ejerciendo su valiosa labor en medio de una realidad adversa en este tiempo, debido a la pandemia en que vivimos actualmente. Es en este contexto en que brindarán sus aportes desde la experiencia en las aulas, producto de todos sus años de experiencia en el área.

El análisis de la información en este estudio, se realizó mediante la organización de la información recolectada en matrices de análisis, donde se digitaron estos datos transcribiéndolos palabra por palabra, creando un archivo donde se logró almacenar toda la información de las entrevistas semiestructuradas que se realizaron. (Bautista, 2011). En este momento de la investigación se hizo una revisión y depuración de las respuestas de los participantes que fueron grabadas, seleccionando aquellas que eran un aporte al estudio, las mismas que luego fueron codificadas y transcritas por el investigador, considerando que, en los aportes realizados por los docentes participantes de la investigación, se obtuvo un mensaje oculto o reservado, el mismo que fue necesario realizar la interpretación de los mismos, lo que permitió ir construyendo una nueva teoría mediante el discernimiento de dicha información. (Bautista 2011).

Los aspectos éticos de la investigación cualitativa, en este estudio, son similares a los aspectos éticos de la investigación cuantitativa, los cuales consisten en la confidencialidad, anonimato, presentado un consentimiento informado o autorización para realizar la investigación en los participantes del estudio. (Hernández y Mendoza, 2018). El consentimiento informado expresa el principio que toda persona no puede ser obligada a participar de ninguna investigación, teniendo en cuenta el respeto por las personas, en el consentimiento informado, los participantes expresan su aceptación para participar en la investigación, para lo cual, deben haber recibido una información previa explicando la finalidad de la investigación y los procedimientos que se realizarán en el estudio, finalmente el participante acepta participar en la investigación expresando su consentimiento sin ninguna coacción.(Delgado 2002). En ese sentido, para aplicar los instrumentos a los docentes de Educación Religiosa de ODEC Piura, tendrá en cuenta la confidencialidad de sus respuestas y el anonimato en la entrevista y entrevista con respuesta fundamentada.

\section{RESULTADOS}

De acuerdo al objetivo: determinar los aspectos desarrollados en el modelo de Tony Booth y Mel Ainscow que se pueden considerar en el desarrollo de una teoría que sustente la educación inclusiva en el área de Educación Religiosa, se propone es el siguiente: 
Figura $\mathrm{N}^{\circ} 01$

\section{ESQUEMA DE MODELO TEÓRICO SOBRE EDUCACIÓN INCLUSIVA EN EL ÁREA DE EDUCACIÓN RELLGIOSA}

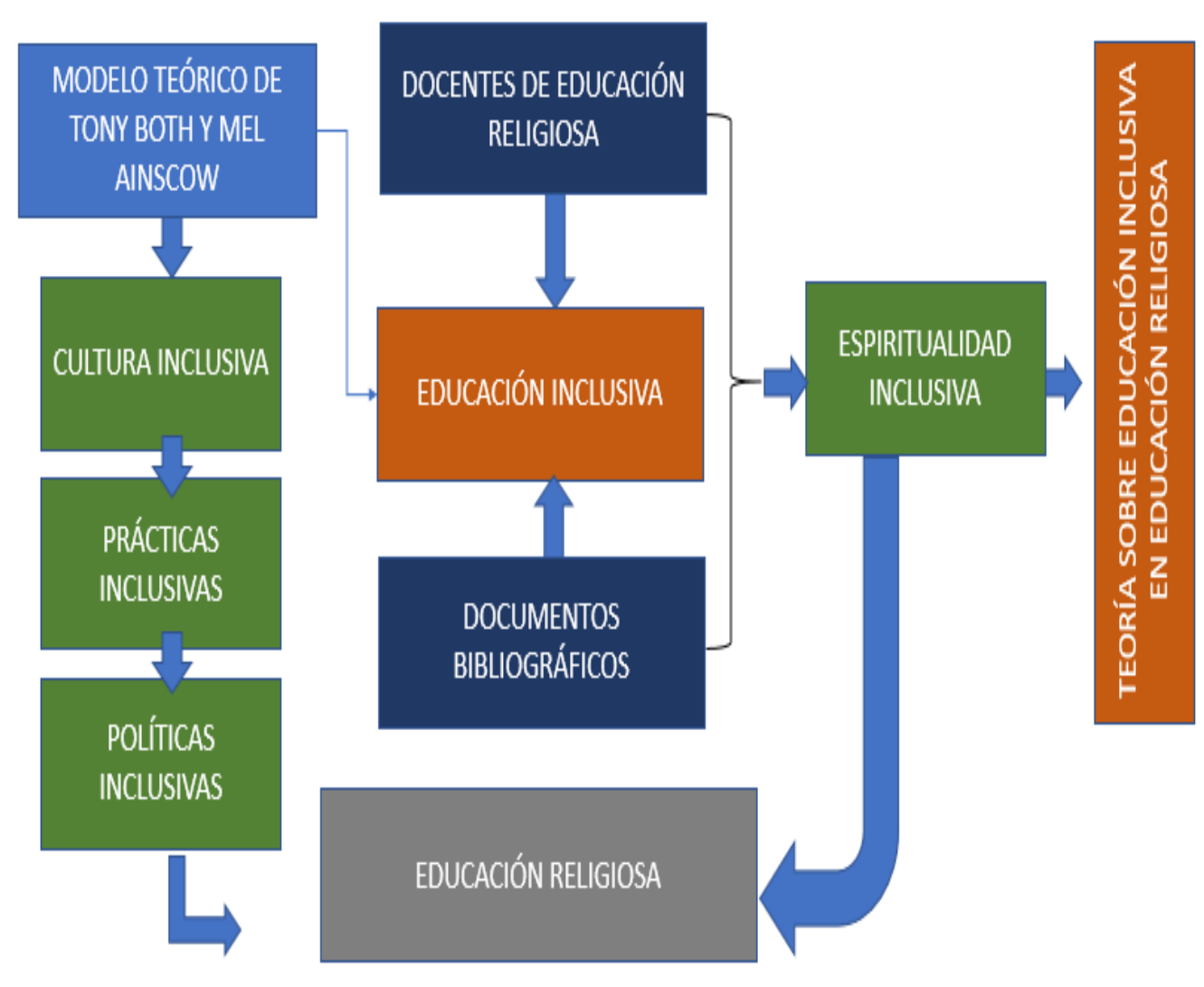

Fuente: creación propia

En el área de Educación Religiosa, los docentes cumplen con su rol inclusivo en concordancia al modelo de Tony Booth y Mel Ainscow, pues, proponen una formación integral, considerando la dimensión espiritual de la persona, así mismo, enriquecen la educación piurana con los valores cristianos que garantizan la realización de una sociedad más justa y solidaria. Dichos valores cristianos están basados en el Evangelio y promueven el respeto de la dignidad de la persona humana, en ese sentido, el área de Educación Religiosa proporciona una gran ayuda a los estudiantes con su formación espiritual, para vivir de acuerdo a las enseñanzas de Jesucristo, creando un ambiente fraterno, donde se ponga en práctica los principios de la Doctrina Social de la Iglesia que inspiran una comunidad en busca del bien común que acoja a todos por igual. (MINEDU 2010).

En relación al rol inclusivos de los docentes del área de Educación Religiosa, éste se expresa con la aceptación de los niños con necesidades educativas especiales, que realizan como parte de su vocación y no como una obligación, haciendo adecuaciones pedagógicas para el proceso de enseñanza aprendizaje y lo que más destaca en esta área es que los docentes imparten el buen trato a los estudiantes, brindándoles 
un acompañamiento personalizado (Informante 2, Matriz $\mathrm{N}^{\circ} 3$ ).

Así mismo, los sistemas de educación inclusiva dentro de las prácticas inclusivas de los docentes de Educación Religiosa se caracterizan por brindar el buen trato a los educandos, la enseñanza del amor al prójimo, que de acuerdo al modelo de Tony Booth y Mel Ainscow garantiza que los estudiantes se valoren a sí mismos y a los demás, sintiéndose amados y acogidos en la escuela. Además, proclamar el amor al prójimo es un elemento esencial para el respeto de la diversidad que se propone en dicho modelo, por tal motivo, los docentes aplican estrategias metodológicas considerando las necesidades educativas de los estudiantes, teniendo en cuenta los diferentes ritmos y estilos de aprendizaje.

En cuanto a los valores inclusivos para crear culturas inclusivas, en el área de Educación Religiosa se practica la participación, pues, los docentes brindan oportunidades a todos los estudiantes para que participen del proceso de enseñanza aprendizaje sin ninguna discriminación, en ese sentido realizan las adaptaciones curriculares, así mismo, la diversidad se respeta en el área de Educación Religiosa, pues, se motiva a la participación solidaria entre los estudiantes, acompañándolos en la valoración del otro, haciendo que se sientan queridos y aceptados entre todos, de la misma manera, el respeto es otro valor que se considera en el área de Educación Religiosa, es elemental en la práctica docente, ya que se considera como parte fundamental para una buena convivencia en el aula y la igualdad. Finalmente, la igualdad es garantizada dentro de la práctica pedagógica del área, a través de diversas estrategias metodológicas, las cuales tienen la finalidad de promover la caridad y la misericordia entre los estudiantes, cimentadas en el principio del Bien común.

Así mismo, en lo que se refiere a las dimensiones del modelo de Tony Booth y Mel Ainscow que son útiles para desarrollar una teoría de la educación inclusiva en el área de Educación Religiosa, se asume la dimensión crear culturas inclusivas, para lo cual, se realiza un trabajo con entusiasmo y alegría de los docentes del área, proclamando la Palabra de Dios a los estudiantes, promoviendo el amor al prójimo y el respeto mutuo, haciendo que todos los estudiantes se sientan queridos, valorados e incluidos en la Institución Educativa. En ese sentido, en el área de Educación Religiosa se promueve una cultura inclusiva mediante la participación de todos los educandos en las diversas sesiones de aprendizaje, para lo cual, aplican diversas estrategias metodológicas, atendiendo las distintas necesidades educativas de todos los estudiantes. Para concretar la dimensión elaborar políticas inclusivas en el área de Educación Religiosa se promueve una escuela para todos aplicando estrategias metodológicas que generen la práctica de valores cristianos como la fraternidad y la caridad, asegurando el trabajo en equipo para que todos los estudiantes logren los aprendizajes sin barreras como la discriminación en el aula. Finalmente, para concretar la dimensión desarrollar culturas inclusivas en esta área, los docentes demuestran que realizan esta dimensión a través de diversas estrategias metodológicas, elaborando sesiones muy didácticas para 
que todos los estudiantes participen, considerando los estilos y ritmos de aprendizaje, atendiendo la diversidad, para lo cual, gestionan sus propios recursos pedagógicos,

La información documental tomada de Lozano, Cerezo y Alcaraz (2015), Martínez, Aquino, Izquierdo y Ramón (2015) y Moreno y Tejada (2018) que complementa los aportes del Modelo de Tony Booth y Mel Ainscow y son útiles para generar una teoría de la educación inclusiva en el área de Educación Religiosa de acuerdo a la sub categoría "Crear culturas inclusivas" es generar un ambiente acogedor e integrador, donde los estudiantes se sientan queridos y valorados para la participación de todos los estudiantes en el proceso de enseñanza aprendizaje, promover valores inclusivos, creando una escuela abierta, participativa e integradora sin discriminación, donde toda la comunidad educativa esté unidad en pro de este objetivo.

Además, para la sub categoría "Elaborar políticas inclusivas" la información documental que complementa los aportes del modelo de Tony Booth y Mel Ainscow para generar una teoría de la educación inclusiva en el área de Educación Religiosa, desde la experiencia de los docentes del área, se desarrollan estrategias que facilita el logro de aprendizajes que contribuyen en su desarrollo personal, quienes realizan planificaciones con adaptaciones curriculares, gracias a la capacitación que reciben.

Finalmente, de acuerdo a la sub categoría "Desarrollar prácticas inclusivas" los aportes documentales que complementan el modelo de Tony Booth y Mel Ainscow que son útiles para desarrollar una teoría de la inclusión en el área de Educación Religiosa son garantizar la participación de los estudiantes en el proceso enseñanza aprendizaje sin discriminación entre los estudiantes, desarrollar los recursos para el logro de los aprendizajes de los estudiantes, rompiendo las barreras educativas, mostrar mayor interés por los estudiantes que presentan ausentismo o abandono escolar, formando estudiantes con una sana autoestima e inteligencia emocional, todo eso gracias a una buena formación profesional y una constante capacitación docente para concretar proyectos educativos institucionales que promuevan la inclusión.

\section{DISCUSIÓN}

En relación al rol inclusivo de los docentes, en el desarrollo del área de Educación Religiosa, se aplica el modelo de Tony Booth y Mel Ainscow eliminando las barreras para el aprendizaje, pues, los docentes, desde la Doctrina Social de la Iglesia, consideran que todos los estudiantes tienen derecho a la educación porque tienen dignidad, pues, son hijos de Dios, es por eso, que no se discriminan las evidencias que presentan, éstas son valoradas y se motiva al estudiante a realizar su mejor esfuerzo de acuerdo a sus habilidades y capacidades. Es así que, en el área de Educación Religiosa se considera la enseñanza para todos los estudiantes con o sin necesidades educativas especiales, tal como se describe en el Índice para la inclusión y el aporte de Ainscow (2003), Barriga, Del Pino y Gonzales (2018) y Ruíz (2016) quienes 
precisan que los alumnos con necesidades educativas especiales pueden recibir una educación en las escuelas normales. Así lo confirma Duran y Martínez (2018), para quien el rol que desempeña el docente inclusivo es comprender las necesidades educativas de los estudiantes sin exclusión de nadie, utilizando diversas estrategias para promover la inclusión.

Referente a los sistemas de educación inclusivos en el área de Educación Religiosa se pone bajo manifiesto la relevancia de la dignidad de la persona humana, pues, de acuerdo a la Doctrina Social de la Iglesia el hombre y la mujer han sido creados a imagen y semejanza de Dios, por lo tanto, estamos llamados a vivir en común unidad con los demás, en un encuentro solidario y fraternal. (Pontificio Consejo "Justicia y Paz". 2005). Además, la Doctrina Social de la Iglesia tiene como principio epistemológico la no discriminación, ya que la Iglesia proclama que todas las personas tienen derechos fundamentales como el derecho a la educación, en ese sentido, no se le puede excluir a ningún alumno del proceso enseñanza aprendizaje, contrarrestando cualquier forma de discriminación, la Doctrina Social de la Iglesia nos hace un llamado a ejercer lazos de amor, de justicia y caridad entre todos. (Pontificio Consejo "Justicia y Paz". 2005). Así mismo, la Doctrina Social de la Iglesia expresa que la educación inclusiva siempre ha sido un tema abordado por la Iglesia, el cual, nos enseña que Dios no hace distinción de nadie, por lo tanto, no debe haber discriminación de nadie, ya que todos somos hijos de Dios. (Pontificio Consejo "Justicia y Paz". 2005)

De acuerdo a los valores inclusivos, todos los elementos que son parte del área de Educación Religiosa están acompañados con los aportes del índice de Booth y Ainscow, quienes expresan que los valores inclusivos son fundamentales para crear una cultura inclusiva, así mismo, se asisten con el aporte de Clavijo y Bautista (2020) quienes explicaron que los valores inclusivos permiten mejorar la calidad educativa en las Instituciones Educativas. Así lo expresa la Doctrina Social de la Iglesia, que señala que el valor de la igualdad es un principio que tiene su esencia en el postulado que todos somos hijos de Dios, por lo tanto, no debe haber diferencias entre los estudiantes por origen, religión, status social, raza o sexo, pues, todos somos miembros del cuerpo de Cristo. (Pontificio Consejo "Justicia y Paz". 2005). En ese sentido, el valor de la participación de los estudiantes para la Doctrina Social de la Iglesia es un elemento clave para lograr que el individuo pueda contribuir a la vida cultural y social, con la participación se puede promover el principio del bien común, el cual, brinda la oportunidad a los más desvalidos o disminuidos de alcanzar su realización, por tal motivo, la participación será un elemento esencial para que los estudiantes con o sin necesidades educativas especiales puedan tener un rol importante en su institución educativa. (Pontificio Consejo "Justicia y Paz". 2005)

Referente a las dimensiones del modelo de Tony Booth y Mel Ainscow crear culturas incluisiva, elaborar políticas inclusivas y desarrollar prácticas inclusivas, el aporte de los docentes de Educación Religiosa se consolida en el Índice para la Inclusión, pues, de acuerdo a dichas dimensiones, en la 
contribución de Booth y Ainscow (2000) encontramos que desarrollar prácticas inclusivas tiene como objetivo asegurar que las actividades escolares promuevan la integración y participación de los estudiantes en su contexto social. considerando que, de acuerdo a la Doctrina Social de la Iglesia, los alumnos tienen dignidad regia, por ser hijos adoptivos de Dios. Además, crear culturas inclusivas en el área de Educación Religiosa se convierte en un elemento que se puede lograr gracias a la práctica del respeto de la dignidad de la persona humana, pues, como lo señala la Doctrina Social de la Iglesia una sociedad podrá ser justa si en ella se considera al otro como un hermano, por eso, nos invita a no discriminar a nadie, comprender que hay personas que merecen que se les proporcionen los medios necesarios para poder vivir con dignidad y en este caso esas personas vienen a ser nuestros alumnos con o sin necesidades educativas especiales. (Pontificio Consejo "Justicia y Paz". 2005). En lo que se refiere a las políticas de inclusión, dentro del área de Educación Religiosa los docentes realizan diversas capacitaciones pedagógicas para aplicarlas, así como concluye Castillo (2015) en su investigación doctoral, señalando que es necesario una buena formación docente para asegurar que los maestros puedan atender a todos los estudiantes con o sin necesidades educativas especiales. Así mismo, concluye Subiria (2019), manifestando que es un desafío atender a los niños con necesidades educativas especiales, para lo cual, se necesita un gran compromiso de los educadores, el cual, los docentes de Educación Religiosa realizan arduamente. Además, todos los aportes de los educadores se centran en los postulados de Booth y Ainscow (2000) quienes refieren que las políticas de inclusión se realizan mediante diversas actividades pedagógicas que puedan aportar a la obtención de la inclusión en la Institución Educativa.

En el análisis documental sobre la dimensión "Crear culturas inclusivas", los aportes de los docentes del área de Educación Religiosa están sustentados por el estudio de Clavijo y Bautista (2020) quien expresa que aplicar modelos de inclusión es fundamental para mejorar los procesos de calidad y construir una Institución Educativa democrática, equitativa y justa. Al respecto, la Doctrina Social de la Iglesia está presente en la convivencia social, la cual, está determinada por la calidad de vida de las personas. La Doctrina Social de la Iglesia establece que todo hombre es único e irrepetible, es un ser predispuesto a la socialización con los demás, es decir, está abierto a convivir con los demás, en una red de familias y grupos sociales, y esta convivencia debe estar caracterizada por relaciones de encuentro, de intercambio, de diálogo y comunicación. (Pontificio Consejo "Justicia y Paz". 2005).

Así mismo, sobre la dimensión "Elaborar políticas inclusivas" en el análisis documental, los aportes de los docentes de Educación Religiosa tienen sustento en el estudio Tesén (2020) que indica que los cambios innovadores de la educación inclusiva dependen de la gestión pedagógica en una Institución Educativa. En esta sub categoría, consideramos la enseñanza de la Doctrina Social de la Iglesia, que precisa que el hombre puede alcanzar una calidad de vida, si se considera el bien común, es decir, buscar 
que todas las personas alcancen satisfacer sus necesidades, sus derechos y lograr sus aspiraciones. (Pontificio Consejo "Justicia y Paz", 2005).

Finalmente, en relación a la dimensión o sub categoría "Desarrollar prácticas inclusivas", también tenemos el aporte de Aguinaga, Velázquez y Rimari (2018) quien es su estudio demuestra la necesidad de realizar proyectos educativos que fortalezcan la educación inclusiva en las Instituciones Educativas. En ese sentido, la Doctrina Social de la Iglesia hace un llamado a los Organismos internacionales para asegurar la igualdad entre todas las personas garantizando el respeto de las diversidades, entonces se concluye que, de acuerdo a la Doctrina Social de la Iglesia, se establece que la exclusión se puede contrarrestar con el principio del destino universal de los bienes, el cual, invita a realizar un mundo solidario y justo, donde todas las personas tengan derecho al bienestar y al desarrollo integral, para que todos puedan gozar de un mundo más humano, donde se valore e integre a todas las personas, jóvenes, mujeres, a los discapacitados, a los migrantes, a los analfabetos, es decir, a los más vulnerables y desposeídos. (Pontificio Consejo "Justicia y Paz". 2005).

\section{CONCLUSIONES}

Los aspectos desarrollados en el modelo de Tony Booth y Mel Ainscow que se han considerado en el desarrollo de una teoría que sustente la educación inclusiva en el área de Educación Religiosa son las categorías: crear culturas inclusivas, elaborar políticas inclusivas y desarrollar prácticas inclusivas.

Las prácticas inclusivas en el área de educación religiosa según el modelo de Tony Booth y Mel Ainscow; se caracterizan por la aceptación de los niños con necesidades educativas especiales, el respeto por el derecho a la educación de todos, donde la inclusión es vista como parte del trabajo del área, y deben hacer adecuaciones a sus programaciones; especialmente, destaca el buen trato que se brinda a estos estudiantes, asistiéndoles con un acompañamiento personalizado, promoviendo el amor al prójimo en sus estudiantes, centrado en las enseñanzas del evangelio.

En cuanto a las dimensiones del modelo de Tony Booth y Mel Ainscow que son útiles para desarrollar una teoría de la educación inclusiva en el área de Educación Religiosa en relación a la dimensión crear culturas inclusivas, encontramos que los docentes de Educación Religiosa construyen una comunidad inclusiva, infunden respeto mutuo, hacen que los estudiantes se sientan queridos, valorados e incluidos, utilizando estrategias metodológicas para desarrollar la inclusión en la escuela. De acuerdo a la segunda dimensión elaborar políticas inclusivas, los docentes de Religión promueven una "escuela para todos" aplicando estrategias metodológicas, promueven la fraternidad y la caridad, promueven el trabajo en equipo para asegurar el aprendizaje y la participación sin discriminación de los estudiantes. En relación a la dimensión desarrollar prácticas inclusivas, los docentes de dicha área realizan diversas estrategias metodológicas para la educación inclusivas, elaboran sesiones didácticas para la 
participación de todos los estudiantes, considerando los estilos y ritmos de los estudiantes, fortalecen la comprensión de la diversidad entre los estudiantes y gestionan sus propios recursos pedagógicos.

La información documental que complementa los aportes del Modelo de Tony Booth y Mel Ainscow y es útil para desarrollar una teoría de la educación inclusiva en el área de Educación Religiosa, en cuanto a la primera sub categoría crear culturas inclusivas de acuerdo a los aportes de Martínez, Aquino, Izquierdo y Ramón. (2015) es generar un ambiente acogedor e integrador, donde los estudiantes se sientan queridos y valorados para la participación de todos en el proceso de enseñanza aprendizaje, asimismo, crear culturas inclusivas es promover valores inclusivos, creando una escuela abierta, participativa e integradora sin discriminación, donde todo toda la comunidad educativa esté unidad en pro de este objetivo. Referente a la segunda sub categoría elaborar políticas inclusivas, Moreno y Tejada. (2018) complementan el trabajo de Tony Booth y Mel Ainscow, cuando postulan que el diseño de políticas inclusivas debe ser consideradas dentro del proyecto educativo de la escuela, es necesario contar con planes flexibles y adaptados a la realidad del estudiante. Además, Martínez, Aquino, Izquierdo y Ramón. (2015)8 complementa el modelo de Tony Booth y Mel Ainscow, señalando que es necesrio realizar una planificación con diversas adaptaciones curriculares para contar con un plan de educación inclusiva a nivel institucional. Así mismo, en la tercera sub categoría, desarrollar prácticas inclusivas Martínez V, Aquino S, Izquierdo J, Ramón P. (2015) manifiestan que es necesario garantizar la participación de los estudiantes sin discriminación en el proceso enseñanza aprendizaje, generar los recursos para que todos los alumnos logren los aprendizajes rompiendo las barreras educativas, mostrar mayor interés por aquellos estudiantes que presentan ausentismo o abandono escolar, lográndose concretar proyectos educativos institucionales que promuevan la inclusión, además, formar estudiantes con una sana autoestima e inteligencia emocional, todo eso gracias a una buena formación profesional así como una constante capacitación docente.

La propuesta de un modelo teórico con teoría fundamentada sobre educación inclusiva en el área de Educación Religiosa a partir del modelo de Tony Booth y Mel Ainscow se expresa de la siguiente manera:

La educación inclusiva desde el área de educación religiosa, se viene entendiendo como la acción que realiza el docente de área, considerando que brinda una formación integral desde la dimensión espiritual de la persona, caracterizándose por la aceptación de los educandos con necesidades educativas especiales y por el buen trato que se brinda a todos los estudiantes, donde un elemento fundamental para cimentar la educación inclusiva en dicha área, son los principios de la Doctrina Social de la Iglesia.

Así mismo, los sistemas de educación inclusiva se reflejan en la enseñanza del amor al prójimo, garantizando que los estudiantes se sientan amados y acogidos en la escuela, proclamando el amor al prójimo. En cuanto a los valores inclusivos en el área de Educación Religiosa, se practica la participación 
sin ninguna discriminación, la diversidad es respetada a través solidaridad ente los estudiantes, siendo el respeto el valor esencial para la labor el área y la igualdad se realiza en la aplicación de estrategias metodológicas como el trabajo en equipo, estos valores están en concordancia con el principio del Bien Común de la Doctrina Social de la Iglesia. En el aspecto de las dimensiones del modelo de Tony Booth y Mel Ainscow que son útiles para desarrollar una teoría de la educación inclusiva en el área de Educación Religiosa, en cuanto a la dimensión crear culturas inclusivas, se proclama el Evangelio promoviendo el amor al prójimo y el respeto de la dignidad de la persona humana, además, se puede comprender que dentro del área se pueden elaborar políticas inclusivas aplicando diversas estrategias metodológicas que contribuyan al trabajo en equipo, donde todos los estudiantes puedan practicar los valores cristianos como la fraternidad y la caridad. Así mismo, para desarrollar prácticas inclusivas, se manifiesta que es importante desarrollar sesiones de aprendizaje adaptadas a las necesidades educativas de todos los estudiantes con o sin necesidades educativas especiales. Estos aportes del área de Educación Religiosa se complementan con el modelo de Tony Booth y Mel Ainscow (2000) y los documentos que son parte de este análisis, los cuales indican que para crear culturas inclusivas se necesita generar un ambiente acogedor e integrador, promoviendo los valores inclusivos para realizar una escuela abierta y participativa sin ninguna discriminación. Finalmente, elaborar políticas inclusivas es ofrecer estrategias dentro del área de Educación Religiosa para que los estudiantes puedan realizar su desarrollo personal. Y referente a desarrollar prácticas inclusivas es preciso garantizar la participación de los estudiantes en el proceso de enseñanza aprendizaje sin discriminación entre los estudiantes, pues, de acuerdo al principio universal de los bienes, los docentes de Educación Religiosa deben fomentar un mundo justo y solidario donde los estudiantes puedan gozar del derecho al bienestar y al desarrollo integral en las Instituciones Educativas. 


\section{REFERENCIAS}

Aguinaga S. Velazquez M. y Rimari M. (2018). Modelo contextualizado de inclusión educativa. https://www.scielo.sa.cr/scielo.php?script=sci_abstract\&pid=S2215-

$26442018000200007 \& \operatorname{lng}=$ en\&nrm=iso\&tlng=es

Barriga Galeano, E., del Pino Tortonda, A. ., \& González Correa, R. de la A. . (2019). La educación inclusiva y sus nexos con la perspectiva de género en la cultura popular contemporánea . Polyphōnía. Revista De Educación Inclusiva / Polyphōnía. Journal of Inclusive Education, 3(1), 120-132. https://revista.celei.cl/index.php/PREI/article/view/272

Bautista N. (2011). Proceso de la investigación cualitativa Epistemología, metodología y aplicaciones. https://www.eesnsrmadrededios.edu.pe/wp-content/uploads/2020/10/Proceso-de-lainvestigacion-cualitativa.pdf

Booth T. y Ainscow M. (2000). Índice de iclusión. https://cupdf.com/document/indice-de-inclusion55c9db3fcb131.html

Castillo C. (2015). La educación inclusiva y lineamientos prospectivos de la formación docente: una visión de futuro. https://revistas.ucr.ac.cr/index.php/aie/article/view/18534

Clavijo R. y Bautista M. (2020). La educación inclusiva. Análisis y reflexiones en la educación superior ecuatoriana. https://alteridad.ups.edu.ec/index.php/alteridad/article/view/1.2020.09/3574

Delgado M. (2002). Aspectos éticos de toda investigación consentimiento informado ¿Puede convertirse la experiencia clínica en investigación científica? https://www.redalyc.org/articulo.oa?id=195118154004

Durán S. y Martínez H. (2018). Rol de epistemología en la inclusión educativa ecuatoriana. https://www.eumed.net/rev/atlante/2018/10/epistemologia-inclusion-educativa.html

Educación inclusiva Piura. (2011). Educación inclusiva Piura. https://educacioninclusivapiura.blogia.com/

Hernández R. \& Mendoza C. (2018). Metodología de la investigación. Las rutas cuantitativa, cualitativa y mixta. https://virtual.cuautitlan.unam.mx/rudics/?p=2612

Jacinto P. (2019). Cultura escolar en educación inclusiva y su influencia en las prácticas inclusivas en las Instituciones Educativas del Nivel Secundario en el Distrito de Pariñas - Piura 2017 https://repositorio.ucv.edu.pe/bitstream/handle/20.500.12692/28858/Jacinto_HPY.pdf?sequence=1 \&isAllowed=y

López, L. (2015). Hacia una escuela inclusiva. Avances En Supervisión Educativa. https://doi.org/10.23824/ase.v0i23.31

Lozano, J., Cerezo, M. C., y Alcaraz, S (2015). Plan de Atención a la diversidad. Tendencias Pedagógicas, 26, 331-332. Recuperado a partir de https://revistas.uam.es/tendenciaspedagogicas/article/view/2190 
Martínez V, Aquino S, Izquierdo J, Ramón P. (2015). Investigación e Innovación en Inclusión Educativa Diagnósticos, Modelos y Propuestas. https://www.researchgate.net/publication/282327052_Investigacion_e_Innovacion_en_Inclusion_E ducativa_Diagnosticos_Modelos_y_Propuestas

MINEDU. (2010). Orientaciones para el trabajo pedagógico. Área de Educación Religiosa. http://www.minedu.gob.pe/minedu/archivos/a/002/03-bibliografia-para-ebr/11-otpreligion2010.pdf Moreno R y Tejada A. (2018). Atención a la Diversidad e Inclusión Educativa: Implicaciones didácticas. https://sid.usal.es/idocs/F8/FDO27377/iAccessibility_15.pdf

Naciones Unidas (ONU). (2018). La agenda 2030 y los objetivos de desarrollo sostenible una oportunidad para América Latina y el Caribe. https://repositorio.cepal.org/bitstream/handle/11362/40155/24/S1801141_es.pdf

Organización de las Naciones Unidas para la Educación, la Ciencia y la Cultura (UNESCO). (2014). Indicadores UNESCO de la cultura para el desarrollo. https://es.unesco.org/creativity/sites/creativity/files/iucd_manual_metodologico_1.pdf

Organización de las Naciones Unidas para la Educación, la Ciencia y la Cultura (UNESCO). (2008). Conferencia Internacional de Educación. http://www.ibe.unesco.org/fileadmin/user_upload/Policy_Dialogue/48th_ICE/G eneral_Presentation-48CIE-4_Spanish_.pdf

$\begin{array}{lccc}\text { PONTIFICIO CONSEJO "JUSTICIA } & \text { Y PAZ". (2005). COMPENDIO DE LA DOCTRINA } \\ \text { SOCIAL } & \text { DE } & \text { LA } & \text { IGLESIA. }\end{array}$
https://www.vatican.va/roman_curia/pontifical_councils/justpeace/documents/rc_pc_justpeace_doc _20060526_compendio-dott-soc_sp.html

Ruiz, P. (2016). Percepciones de docentes y padres sobre la educación inclusiva y las barreras para su implementación en Lima, Perú. Revista Latinoamericana de Inclusión Educativa,. http://repositori.uji.es/xmlui/handle/10234/166705

Subiria M. (2019) Inclusión educativa en la Institución Educativa No 1213 - Red 11 - Ugel 06 Ate 2109 https://repositorio.ucv.edu.pe/bitstream/handle/20.500.12692/40188/Subiria_ME.pdf?sequence=1\& isAllowed $=\mathrm{y}$

Tesén R. (2020). "Herramientas tecnológicas y el aprendizaje basado en la investigación: una perspectiva para desarrollar la educación inclusiva". https://www.researchgate.net/publication/340087705_Herramientas_Tecnologicas_y_el_Aprendiza je_Basado_en_la_Investigacion_Una_perspectiva_para_desarrollar_la_Educacion_Inclusiva

Vásquez J. (2017). Modelo de Gestión Index para la mejora de la Educación Inclusiva en las instituciones educativas públicas del Distrito de Chiclayo - 2016. https://repositorio.ucv.edu.pe/handle/20.500.12692/2309 